\title{
An Efficient Delay Estimation Model for High Speed VLSI Interconnects
}

\author{
M. KAVICHARAN \\ Dept. of ECE \\ National Institute of \\ Technology \\ Warangal, INDIA
}

\author{
N.S. MURTHY \\ Dept. of ECE \\ National Institute of \\ Technology \\ Warangal, INDIA
}

\author{
N. BHEEMA RAO \\ Dept. of ECE \\ National Institute of \\ Technology \\ Warangal, INDIA
}

\begin{abstract}
In this paper a closed-form matrix rational model for the computation of step and finite ramp responses of Resistance Inductance Capacitance (RLC) interconnects in VLSI circuits is presented. This model allows the numerical estimation of delay and overshoot in lossy VLSI interconnects. The proposed method is based on the U-transform, which provides rational function approximation for obtaining passive interconnect model. With the reduced order lossy interconnect transfer function, step and finite ramp responses are obtained and line delay and signal overshoot are estimated. The estimated delay and overshoot values are compared with the Euder method, Pade method and HSPICE W- element model. The 50\% delay results are in good agreement with those of HSPICE within $0.5 \%$ error while the overshoot error is within $1 \%$ for a $2 \mathrm{~mm}$ long interconnect. For global lines of length more than $5 \mathrm{~mm}$ in SOC (system on chip) applications, the proposed method is found to be nearly four times more accurate than existing methods.
\end{abstract}

Keywords: Delay; matrix rational model; ramp input; RLC interconnects; transient analysis; transfer function; Uapproximation.

Received: June 30, 2021. Revised: December 5, 2021. Accepted: December 19, 2021. Published: January 8, 2022.

\section{Introduction}

As the physical dimensions in VLSI technologies scale down, interconnect delay dominates the gate delay in determining circuit performance [1]. In deep submicron VLSI circuits it is necessary to have computationally economical and accurate interconnect delay models. Thus for the design of complex circuits, more accurate analytic models are needed to predict the interconnect delay accurately.

Originally VLSI interconnects were modeled as RC lines and single pole Elmore-based models [2]-[3] because of long channel device delay dominance over negligible interconnect delay. However for high speed interconnects, inductance effects are becoming progressively important and can no longer be ignored. Under these circumstances, the Elmore model fails since it does not consider the inductance effects [4]. It is necessary to use a second-order model, which includes the effect of inductance. Kahng et al. considered equivalent Elmore delay model based on the Resistance Inductance and Capacitance (RLC) of the interconnects [4] and [5]. Ismail et al. [6] proposed two pole model to capture far end time domain solution for single line interconnect.

A simplified voltage transfer function obtained using Taylor series approximation for transient analysis [7]-[8] has less accuracy in delay calculation. Nakhla et al.[9] use modified nodal analysis (MNA) for obtaining far end and near end responses of interconnects. Roy [10] extended [9] for obtaining more accurate far end responses of coupled RLC interconnects using delay algebraic equations.
A matrix rational-approximation model for SPICE analysis of high-speed interconnects is presented in [11]-[12], however, the approximations made to derive the models contributed to inaccuracy. This has been extended using Pade approximation model [13] to estimate the delay of interconnects. All the above models still suffer from various inaccuracies and need improvement for accurate delay estimations.

In this paper, we present an improved analytic delay model by extending the concepts developed in [11]-[13] for on-chip RLC interconnects. The accuracy of Euder approximation method [16] has been improved by using fourth order MacLaurin series for RLC interconnects and compared with Pade method, proposed method and HSPICE. The proposed model is based on U-transform [14]-[15], which is simple in structure and easier to implement. For a given number of terms used in the transform, the U-approximant requires less algebraic manipulations than the Pade scheme and thus computationally less expensive. This U-transform is used to solve the Telegraphers equation solution for the first time.

The remainder of the paper is organized as follows. Section II briefly describes the mathematical analysis to determine the linear transfer function of RLC interconnect to find the transient analysis. Section III develops the proposed U-model for single RLC line. For validation of the proposed model simulation results are compared with standard HSPICE and reported in sections IV. Conclusions and future scope appear at the end. 


\section{Analysis of RLC Interconnect}

The solution of interconnects are described by telegrapher's equations as

$$
\begin{aligned}
& \frac{\partial}{\partial x} V(z, s)=-(R+s L) I(z, s) \\
& \frac{\partial}{\partial x} I(z, s)=-s C V(z, s)
\end{aligned}
$$

where ' $s$ ' is the Laplace-transform variable, $z$ is a variable which represents position; $V(z, s)$ and $I(z, s)$ stand for the voltage and current vectors of the transmission line, respectively, in the frequency domain; and $\mathrm{R}, \mathrm{L}$ and $\mathrm{C}$ are the per unit length (p.u.1.) resistance, inductance, and capacitance matrices, respectively.

The solution of (1) can be written as an exponential matrix function as

$$
\left[\begin{array}{c}
V(d, s) \\
-I(d, s)
\end{array}\right]=e^{\phi d}\left[\begin{array}{c}
V(0, s) \\
I(0, s)
\end{array}\right]
$$

where

$$
\phi=\left[\begin{array}{cc}
0 & -Z \\
-Y & 0
\end{array}\right]
$$

and ' $\mathrm{d}$ ' is the length of the transmission line, with $\mathrm{Z}=\mathrm{R}+\mathrm{sL}$ and $\mathrm{Y}=\mathrm{sC}$. The exponential matrix of (2) can be written in terms of cosh and sinh functions as

$$
e^{\phi d}=\left[\begin{array}{cc}
\cosh (d \sqrt{Z Y}) & -Y_{0}^{-1} \sinh (d \sqrt{Y Z}) \\
-Y_{0} \sinh (d \sqrt{Y Z}) & \cosh (d \sqrt{Y Z})
\end{array}\right]
$$

where

$$
Y_{0}=Y(\sqrt{Y Z})^{-1}
$$

Equation (2) does not have a direct representation in the time domain, so it is difficult to analytically predict the delay and overshoot of transmission lines.

\section{Proposed Umodel}

This model is based on a generalized U-transform [14] For the power series expansion of a function $f(x)$, where ' $x$ ' is a complex variable

$$
f(x)=\sum_{n=0}^{\infty} a_{n} x^{n}
$$

The sequence $\left\{s_{n}\right\}$ is a partial sum of original series

$$
s_{n}=\sum_{k=0}^{n-1} a_{k} x^{k}
$$

The closed form rational function approximation for an exponential matrix is

$$
u_{k n}\left(\left\{S_{n}\right\}\right)=\frac{\sum_{j=0}^{n+k-1} x^{i} \sum_{j=0}^{k} w_{k n j} a_{j-i}}{\sum_{j=0}^{k} w_{k n j} x^{i}}
$$

where

$$
w_{k n j}=(-1)^{j} \frac{k !}{j !(k-j) !} \frac{(n+k-j)^{k-2}}{a_{n+k-j-1}}
$$

Thus $u_{\mathrm{kn}}$ represents a table of rational functions, each element of which is obtained from $n+k$ terms of the original sequence $\left\{\mathrm{S}_{\mathrm{n}}, \mathrm{n}=1,2, \ldots\right\}$ and is an approximant of the function $\mathrm{f}(\mathrm{x})$ specified above.

Calculation procedure for estimating delay and overshoot using U-approximants are as follows.

(i) Use the Interconnect line parameters as per Table I.

(ii) Telegrapher's equations are solved and the solution can be written as exponential matrix.

(iii) This transfer function matrix parameters can be approximated using the U-model.

(iv) In the proposed model calculate the coefficient of the exponential function i.e., $\mathrm{a}_{\mathrm{i}}$ where

$$
0 \leq i \leq n
$$

(v) Calculate $\mathrm{w}_{\mathrm{knj}}$ from the relation (4)

(vi) Calculate the inner sum of the Eq (3) numerator.

(vii) Total sum of the numerator is obtained

(viii) Calculate the total sum of the denominator of the Uapproximants

(ix) Calculate the U-approximants

(x) Make use of the U-approximants to get approximated transfer function

(xi) Find the time domain response of approximated transfer function using inverse Laplace transform to estimate delay and overshoot of interconnect.

The basic idea of the matrix rational-approximation model is to use predetermined coefficients to analytically obtain rational functions for (2). To obtain a passive model, the exponential function $\mathrm{e}^{\Phi \mathrm{d}}$ is approximated using $\mathrm{Eq}(3)$ and the resultant model is used for obtaining time response.

A single RLC line is shown in Fig. 1. The line is driven by a step input and $1-\mathrm{V}$ finite ramp with rise time of $0.1 \mathrm{~ns}$. This represents a point-to-point interconnection driven by a transistor (modelled as a resistance $\mathrm{R}_{\mathrm{s}}$ ) and connected to the next gate (modelled as a capacitance $\mathrm{C}_{1}$ ). 


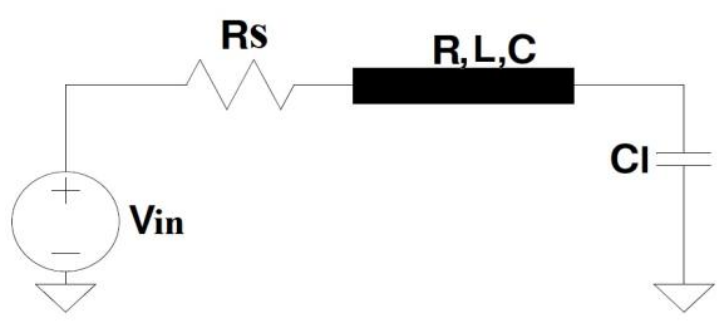

Fig. 1. Circuit model of the single-line distributed RLC interconnect.

The frequency-domain solution at the far end is expressed as

$V_{f}=\frac{V_{\text {in }}}{\left(1+s R_{s} C_{l}\right) \cosh (\Gamma d)+\left(R_{s} Y_{0}+s C_{l} Y_{0}^{-1}\right) \sinh (\Gamma d)}$

where

$$
\Gamma=\sqrt{Y Z}
$$

$R_{s}$ is the source resistance at the near end, $C_{1}$ is the load capacitance at the far end, and $V_{\text {in }}$ is the input voltage. The exact transfer function of distributed RLC transmission line has cosh and sinh terms, which are multiplied with $\mathrm{Y}_{\mathrm{o}}$ and it's inverse. It is extremely difficult to find the time domain response of this complex transfer function, so several approximations are proposed in literature to find the time domain response. An approximate transfer function has been derived using U-transform. This transfer function is inverse Laplace transformed to get time domain response for estimation of delay and overshoot in single RLC interconnect.

\section{Simulation Results}

The single RLC line is presented in this section to demonstrate the validity and efficiency of the proposed method. The results were obtained using MATLAB R2010a operating on HP 64-bit Intel i5 processor with clock speed of $2.53 \mathrm{GHz}$ and are also compared with HSPICE using the Welement method.

The typical interconnect parameters [13] considered for simulation of single RLC interconnect are given in table-I. The Pade approximation, Eudes model and proposed Uapproximation are implemented in MATLAB for the same set of input parameters and various approximation orders.

Table I: The values of Interconnects parameters [13]

\begin{tabular}{|l|l|}
\hline Vdd & $1 \mathrm{v}$ \\
\hline Length & $0.2 \mathrm{~cm}$ \\
\hline Resistance & $88.29 \Omega / \mathrm{cm}$ \\
\hline Capacitance & $1.8 \mathrm{pF} / \mathrm{cm}$ \\
\hline Inductance & $15.38 \mathrm{nH} / \mathrm{cm}$ \\
\hline Load capacitance & $0.05 \mathrm{fF}$ to $0.1 \mathrm{fF}$ \\
\hline Source resistance & $20 \Omega$ to $100 \Omega$ \\
\hline Input ramp rise time & $0.1 \mathrm{~ns}$ \\
\hline
\end{tabular}

The accuracy of proposed model validated using the frequency response of cosh function as shown in Fig. 2. The frequency response is obtained using pade (3/3) and proposed U-model (3/3) are compared with the exact solution of telegrapher's equations. It is observed that, the proposed method is better than Pade method and well matches with exact cosh function for the order of $3 / 3$ up to the frequency of $25 \mathrm{GHz}$.

The far-end responses to a finite ramp input of single interconnect is plotted in Fig. 3. The plots compared the responses of proposed, Pade and HSPICE W-element models.

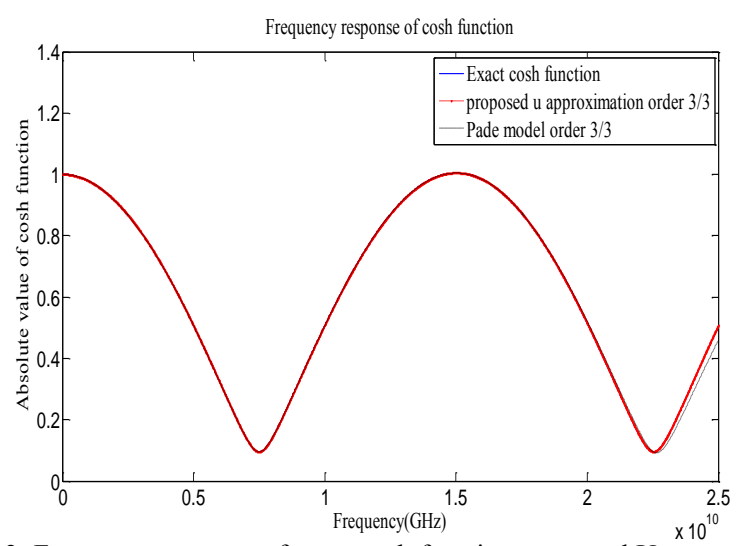

Fig: 2. Frequency response of exact cosh function, proposed $U$-approximation order 3/3 and Pade approximation order 3/3.

From Fig. 3, it is noticed that, the proposed Uapproximation and Pade method are very close as compared to HSPICE. But Eudes model of order 4 has more overshoot as compared to other methods.

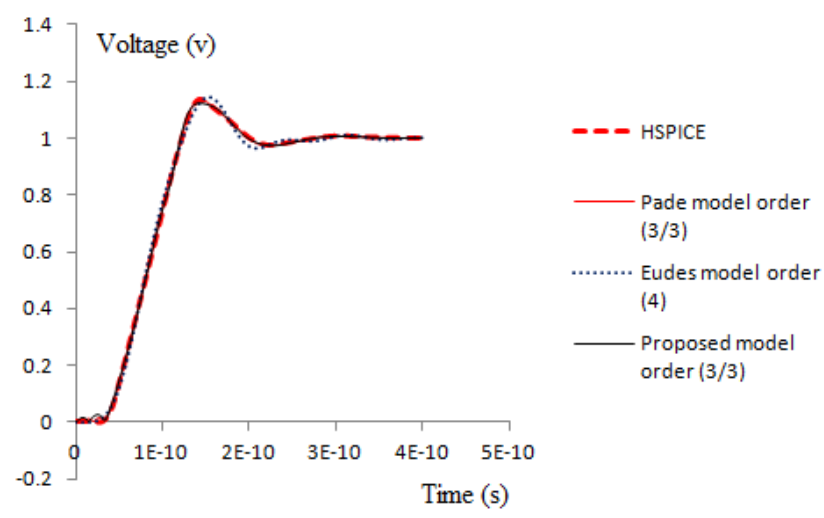

Fig: 3.Transient analysis of single interconnect line, when length $=0.2 \mathrm{~cm}, \mathrm{R}_{\mathrm{s}}=50 \Omega$ and $\mathrm{C}_{\mathrm{l}}=50 \mathrm{fF}$.

The MATLAB results of step response and finite ramp response are plotted for the line length of $0.2 \mathrm{~cm}$, source resistance of $100 \Omega$ and load capacitance of $100 \mathrm{fF}$. Step response in Fig. 4 has less ringing in proposed method as compared to Pade method, for the same approximation order of 3/3, whereas Fig. 5 gives finite ramp response of single line interconnect using U-model matches very well with the 
HSPICE. But Eudes model needs more settling time as compared to the proposed model.

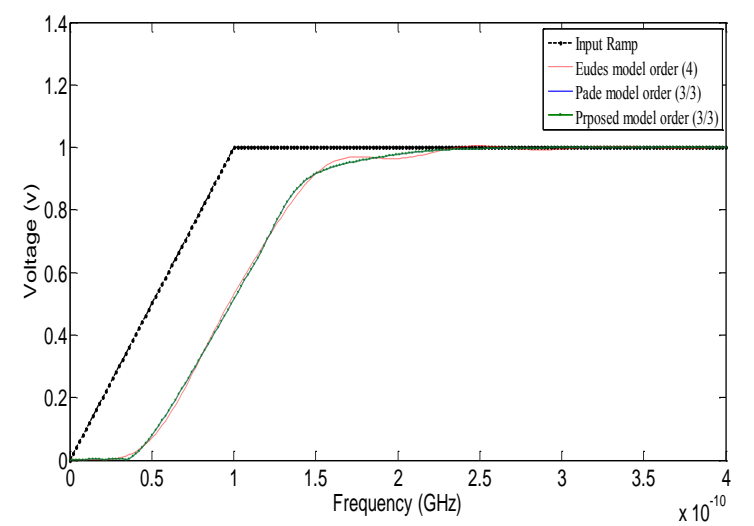

Fig: 4.Step response of single line when length $=0.2 \mathrm{~cm}, \mathrm{Rs}=100 \Omega$ and $\mathrm{Cl}=100 \mathrm{fF}$.

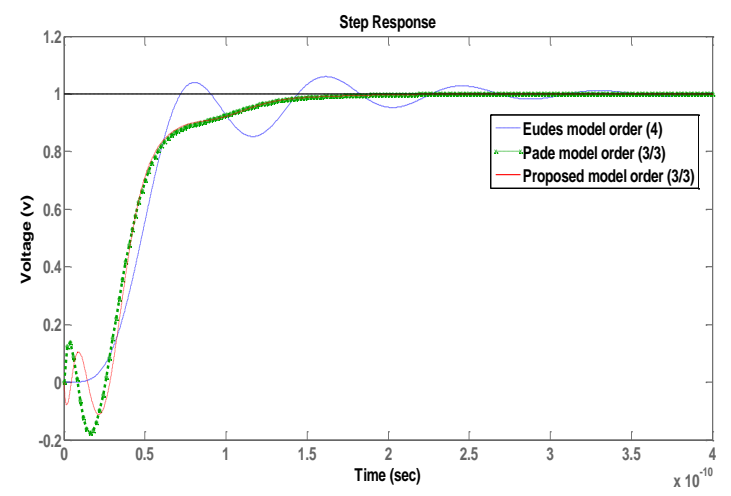

Fig: 5. Ramp response of single line when length $=0.2 \mathrm{~cm}, \mathrm{R}_{\mathrm{s}}=100 \Omega, \mathrm{C}_{\mathrm{l}}=100 \mathrm{fF}$.

Table II: Comparisons of 50\% delay of HSPICE W Element, Eudes model Pade model and proposed model for various lengths, source Resistances and load Capacitances.

\begin{tabular}{|c|c|c|c|c|c|c|}
\hline \multirow{2}{*}{$\begin{array}{c}\mathrm{L} \\
(\mathrm{cm})\end{array}$} & $\begin{array}{c}\text { Rs } \\
(\Omega)\end{array}$ & $\begin{array}{c}\mathrm{Cl} \\
(\mathrm{fF})\end{array}$ & HSPICE & $\begin{array}{c}\text { Eudes model order } \\
(4)\end{array}$ & $\begin{array}{c}\text { Pade model order } \\
3 / 3\end{array}$ & $\begin{array}{c}\text { Proposed } \\
\text { Model order }(3 / 3)\end{array}$ \\
\cline { 3 - 7 } & & & $50 \%$ delay $(\mathrm{ps})$ & $\begin{array}{c}50 \% \text { delay }(\mathrm{ps}) \\
(\% \text { Error })\end{array}$ & $\begin{array}{c}50 \% \text { delay }(\mathrm{ps}) \\
(\% \text { Error) }\end{array}$ & $\begin{array}{c}50 \% \text { delay }(\mathrm{ps}) \\
(\% \text { Error })\end{array}$ \\
\hline \multirow{2}{*}{0.2} & 50 & 50 & 79.8 & $79.1(0.8 \%)$ & $80.2(0.5 \%)$ & $80.2(0.5 \%)$ \\
\cline { 2 - 7 } & 100 & 100 & 98.7 & $96.8(1.92 \%)$ & $98.6(.1 \%)$ & $98.65(0.05 \%)$ \\
\hline \multirow{2}{*}{0.5} & 50 & 50 & 135.7 & $142.8(5.23 \%)$ & $137.8(1.54 \%)$ & $137.7(1.4 \%)$ \\
\cline { 2 - 7 } & 100 & 100 & 156.6 & $162.6(3.83 \%)$ & $151.9(3 \%)$ & $155.3(0.83 \%)$ \\
\hline \multirow{2}{*}{1.0} & 50 & 50 & 231.2 & $250.2(8.21 \%)$ & $211.1(8.69 \%)$ & $224.7(2.811 \%)$ \\
\cline { 2 - 7 } & 100 & 100 & 255.6 & $284.8(11.42 \%)$ & $249.7(2.3 \%)$ & $252.5(1.21 \%)$ \\
\hline
\end{tabular}

Table III: Comparisons of overshoot of HSPICE W Element, Eudes model, Pade model and proposed model for various lengths, source Resistances and load Capacitances.

\begin{tabular}{|c|c|c|c|c|c|c|}
\hline $\begin{array}{c}\mathrm{L} \\
(\mathrm{cm})\end{array}$ & $\begin{array}{c}\text { Rs } \\
(\Omega)\end{array}$ & $\begin{array}{c}\mathrm{Cl} \\
(\mathrm{fF})\end{array}$ & HSPICE & $\begin{array}{c}\text { Eudes model order } \\
(4)\end{array}$ & Pade Order 3/3 & $\begin{array}{c}\text { Proposed } \\
\text { Model order (3/3) }\end{array}$ \\
\cline { 4 - 7 } & & & Overshoot (V) & $\begin{array}{c}\text { Overshoot (V) } \\
(\% \text { Error) }\end{array}$ & $\begin{array}{c}\text { Overshoot (V) } \\
(\% \text { Error) }\end{array}$ & $\begin{array}{c}\text { Overshoot (V) } \\
(\% \text { Error) }\end{array}$ \\
\hline \multirow{2}{*}{0.2} & 50 & 50 & 1.14 & $1.14(0 \%)$ & $1.12(1.7 \%)$ & $1.13(0.87 \%)$ \\
\cline { 2 - 7 } & 100 & 100 & 1.00 & $1.00(0 \%)$ & $1.00(0 \%)$ & $1.00(0 \%)$ \\
\hline \multirow{2}{*}{0.5} & 50 & 50 & 1.15 & $1.24(7.8 \%)$ & $1.15(0 \%)$ & $1.14(0.87 \%)$ \\
\cline { 2 - 7 } & 100 & 100 & 1.00 & $1.03(3 \%)$ & $1.00(0 \%)$ & $1.00(0 \%)$ \\
\cline { 2 - 7 } 1.0 & 50 & 50 & 1.00 & $1.09(9 \%)$ & $1.02(2 \%)$ & $1.01(1 \%)$ \\
\hline
\end{tabular}

The Tables II and III give the comparisons of 50\% delay and overshoot values obtained using HSPICE for various lengths, source Resistances and load Capacitances. These tables include the percentage error values with respect to HSPICE. From Table II the Eudes model of order 4 has worst case error of $11.42 \%$, whereas Pade and proposed models have $8.69 \%$ and $2.811 \%$.

It can be observed that the methods implemented for global lines have more error percentage than our proposed method. Both Pade and proposed methods perform similarly for smaller length interconnects while Eudes method has more error percentage.

As noticed in Table III, the Eudes model has worst case overshoot error percentage of 9\%, but Pade model has an error percentage up to $2 \%$ while the proposed model has error within $1 \%$. In the case of overshoot estimation our model is best for all cases. For $2 \mathrm{~mm}$ range lines the proposed method has delay and overshoot errors within $1 \%$. 


\section{Conclusion}

This paper presents a U-transform based closed form model for delay and overshoot estimation of high speed VLSI interconnects in DSM regime. A single line interconnect has been used for validating the proposed model by comparing with the Eudes model, Pade method and HSPICE. In SOC (system on chip) applications, for global lines of lengths $2 \mathrm{~mm}$ and above the proposed method is found to be more accurate than existing methods. This method can be used to estimate the signal integrity characteristics of Carbon nano tubes.

\section{References}

[1] Semiconductor Industry Association. The International Technology Roadmap for Semiconductors. 1999.

[2] W. C. Elmore, "The transient response of damped linear networks with particular regard to wideband amplifiers,'J. Appl. Phys., vol. 19, no.1, pp. 55-63, Jan. 1948.

[3] T.Sakurai, "Closed-form expressions for interconnection delay, coupling, and crosstalk in VLSI's,"IEEE Trans. Electron Devices, vol. 40, no. 1, pp. 118-124, Jan. 1993.

[4] Kahng A B, Muddu S, "An analytical delay for RLC interconnects", IEEE Trans. Computer-Aided Design of Integrated Circuits and Systems, 1997, 16(12): 1507-1514

[5] Kahng A B, Muddu S, "Two-pole analysis of interconnection trees", Proc. of IEEE MCMC Conf.(MCMC-95), Santa Cruz, California, USA: 1995, 105-110.

[6] Xiaopeng Ji, Long Ge, Zhiquan Wang, "Analysis of on-chip distributed interconnects based on Pade expansion", Journal of Control Theory and Applications, 2009, 7 (1) pp. 92-96.

[7] Ren Yinglei, Mao Junfa and Li Xiaochun, "Analytical delay models for RLC interconnects under ramp input", Frontiers of Electrical and Electronic Engineering in China, vol. 2, pp. 88-91, March 2006.

[8] Y. I. Ismail, E. G. Friedman, and J. L. Neves, "Equivalent Elmore delay for RLC trees," IEEE Trans. Comput.-Aided Design Integr. Circuits Syst.,vol. 19, no. 1, pp. 83-97, Jan. 2000.

[9] N.Nakhla,A.Dounavis,R.Achar,M.Nakhla,"DEPACT:Delay Extraction BasedPassive Compact Transmission-Line Macromodeling Algorithm," IEEE Transactions on Advanced Packaging, vol. 28, issue 1, pp. 13-23, Feb 2005.

[10] S.Roy, A. Dounavis, "Efficient Delay and Crosstalk Modeling of RLC Interconnects using Delay Algebraic Equations", IEEE Transactions on Very Large Scale Integration Systems, vol. 2, issue 2, pp. 342-345, Feb. 2011.

[11] A. Dounavis, R. Achar, and L. Xin,"Passive closed-form transmissionline model for general-purpose circuit simulators," IEEE Trans. Microw. Theory Tech., vol. 47, no. 12, pp. 2450-2459, Dec. 1999.

[12] A. Dounavis, R. Achar, and M. Nakhla, "A general class of passive macromodels for lossy multiconductor transmission lines,'IEEE Trans. Microw. Theory Tech., vol. 49, no. 10, pp. 1686-1696, Oct. 2001.

[13] S. Roy and A. Dounavis, "Closed-Form Delay and Crosstalk Models for RLC On-Chip Interconnects using a Matrix Rational Approximation", IEEE Transactions on Computer-Aided Design of Integrated Circuits and Systems, vol. 28, issue 10, pp. 1481 - 1492, Oct.2009.

[14] Roy, D., Bhattacharya, R., and Bhowmick, S., "Rational approximants generated by the U-transform,"Comput. Phys. Commun. 78, $29-54$ (1993).

[15] Tarun Kumar Sheel, Thesis "Rational approximants generated by Pade approximation and U-transform" University of Dhaka, Bangladesh MARCH 1997.

[16] T.Eudes, B. Ravelo, and A. Louis, "Transient response characterization of the high-speed interconnection RLCG-model for the signal integrity analysis," PIER Journal, Vol. 112, pp. 183-197, 2011.

\section{Creative Commons Attribution License 4.0 (Attribution 4.0 International, CC BY 4.0)}

This article is published under the terms of the Creative Commons Attribution License 4.0 https://creativecommons.org/licenses/by/4.0/deed.en_US 